\title{
Peripherally inserted central catheters: spreading the MAGIC beyond Michigan
}

\author{
Peter Wilson, ${ }^{1}$ Andrew Rhodes ${ }^{2}$
}

\begin{abstract}
'Microbiology \& Virology, University College London Hospitals NHS Foundation Trust, London, UK

${ }^{2}$ Department of Critical Care and Diagnostics, St George's University Hospitals NHS Foundation Trust, London, UK
\end{abstract}

\section{Correspondence to}

Prof Peter Wilson, University College London Hospitals NHS Foundation Trust, London NW1 2PG,UK; peter.wilson9@nhs.net

Accepted 9 June 2021

\section{Linked}

- http://dx.doi.org/10.1136/ bmjqs-2021-013015

\section{Check for updates}

(C) Author(s) (or their employer(s)) 2021. No commercial re-use. See rights and permissions. Published by BMJ.

To cite: Wilson P, Rhodes A BMJ Qual Saf Epub ahead of print: [please include Day Month Year]. doi:10.1136/ bmjqs-2021-013456
Central venous access catheters are often used for patients requiring repeated infusions, blood sampling, invasive monitoring or where peripheral access is difficult. For intravenous access of up to 2 weeks in duration, a midline catheter is usually satisfactory, but for longer-term use, peripherally inserted central catheters (PICCs) are increasingly used, including in clinical areas outside of critical care. ${ }^{1}$

Despite enabling complex care to be delivered more easily, these catheters are associated with significant complications-including central line associated bloodstream infections (CLABSI), venous thromboembolism (VTE) and line occlusion. Such complications can result in poor patient outcomes and are associated with prolonged hospital stays and increased costs of care. In one series of 438 patients with central lines, $61 \%$ reported at least one complication, including bloodstream infection. ${ }^{2}$ Accumulating evidence suggests that being selective about who receives PICC lines, of what type and for what duration, could reduce the frequency of such complications. For example, the use of multilumen PICC lines when a single lumen would suffice is associated with increased early infection and thrombosis. ${ }^{3}$ In a Canadian study, CLABSI was reported in $5.0 \%$ of 907 double-lumen catheters compared with $2.4 \%$ of 618 single lumen catheters. ${ }^{3}$ Thrombosis leading to reinsertion was also much higher with double-lumen lines. ${ }^{3}$ A simple change in practice of inserting single lumen devices, unless there was clear justification for the contrary, may therefore result in reductions in complications as well as major cost savings.

Concurrently, evidence about best practices in implementing interventions to reduce central-line associated complications is also emerging. Critical care units in Michigan, USA, have long demonstrated leadership in research to reduce catheter-related complications. In 2004, Pronovost and colleagues used a package of evidence-based interventions in 108 intensive care units (ICUs) with the aim of reducing CLABSI, including infections associated with PICC lines. ${ }^{4}$ A significant reduction was maintained throughout the 18 months of the study. A key element was the designation of a physician and a nurse as team leaders at each site. This inspired a UK project called Matching Michigan that demonstrated, in a stepped-wedge study design, that a combination of technical and behavioural interventions could lead to significant reductions in CLABSI rates in the critical care setting. ${ }^{5}$ However, these programmes have been limited to the critical care setting, ${ }^{6}$ can be labour-intensive and the long-term effects are uncertain. ${ }^{7}$ While simpler-toimplement educational programmes have been associated with marked reductions in the rate of CLABSI, ${ }^{8}$ training and care processes need to be refreshed regularly and supported with feedback on performance to be effective. ${ }^{9}$

PICCs are now widely used, including in outpatient settings, and complications other than infection are more common than for other central venous catheters. A broader approach, applicable outside of critical care and for a wider set of complications, is therefore needed.

Enter the Michigan Appropriateness Guide for Intravenous Catheters ('MAGIC'), which was developed to determine when PICC insertion is appropriate, with the aim of reducing the incidence of the associated complications. ${ }^{1011}$ Specifically, the major focus of MAGIC was to reduce the use of PICCs for less than 5 days or with multiple lumens and to avoid use in patients with chronic kidney 
disease. ${ }^{11} 12$ Implementation of MAGIC now includes online learning, access to subject matter experts, quarterly knowledge-sharing meetings, feedback on performance and pay-for-performance incentives. ${ }^{11} 12$ Initial research demonstrated preliminary evidence of effectiveness, reducing inappropriate PICC use by $13.8 \%$ in one hospital compared with nine control sites (OR $0.86 ; 95 \%$ CI 0.74 to 0.99 ). PICC line usage decreased and single lumen line use increased, but, although catheter occlusion fell significantly, VTE and CLABSI were not significantly reduced in this relatively small scale study ${ }_{1}^{11}$ and until now, the impact on patient outcomes in a large number of patients across a range of hospitals had not been explored.

It is in this context that a further study in this issue ${ }^{12}$ now builds on this work by demonstrating that the MAGIC programme also reduces the incidence of complications. Data were extracted from case records on PICC insertions across 52 hospitals, with inappropriate use more common in seriously ill patients and CLABSI more common in those judged 'inappropriate'. Following the implementation of MAGIC, catheter occlusion fell from $10.6 \%$ to $7.4 \%$ of PICC lines, VTE from $3.3 \%$ to $2.5 \%$ and CLABSI from $1.8 \%$ to $1.4 \%$. While the study design does not allow causation to be proven, these effects were statistically significant, suggesting the programme to be effective, and that it should be implemented elsewhere. This therefore raises the question of whether the same benefits are likely to be realised elsewhere.

It is clear that inappropriate selection of both patients and intravenous catheter types is an expensive and widespread international problem. In France, when pharmacist approval was required for placement of PICCs, $5 \%$ of requests were declined as being inappropriate. ${ }^{13}$ In Canada, application of the MAGIC definitions suggested $13 \%-21 \%$ of 3479 PICC placements to be inappropriate. ${ }^{14}$ The MAGIC programme, or similar initiatives, are therefore likely to minimise harm to patients from complications associated with inappropriate use of PICCs in other countries. Clear evidence-based criteria for selection of patients for PICC insertion, combined with multidisciplinary communication, as proposed in the MAGIC programme, are likely to be important internationally in deciding when to place a PICC rather than another access device. However, adaptation to local methods and contexts of healthcare delivery are likely to be needed; this is for several reasons. First, countries outside the USA may not share the pay-forperformance framework that provided incentives to hospitals participating in the MAGIC project.

Second, some countries may have already introduced other interventions to reduce complications of central venous catheters. In India, basic training in hand hygiene and hub care, repeated until a high level of knowledge was demonstrated in tests, resulted in CLABSI reduction from 8.7 to $4.5 / 1000$ catheter days. ${ }^{13}$ In Italy, training of healthcare workers based on a package of evidence-based preventative measures including removal of catheters when no longer needed, reduced CLABSI from 8.6 to 2.6/1000 catheter days. ${ }^{14}$

Third, the method of delivery of PICC insertion may vary by country, which has important implications for whom to target with the MAGIC intervention. Delivery of PICC insertion in a number of countries, as in USA, follows a variety of models, including specialist nurse training and delivery, off-site insertion or use of interventional radiology vascular access teams. ${ }^{15}$ Selection of patients and insertion of central venous catheters, particularly outside critical care, are often the responsibility of a dedicated specialist team with access to ultrasound or interventional radiology. High levels of training improve standards of insertion and reduce infection rates, especially when combined with surveillance and feedback of performance. ${ }^{4} 5$ However, dedicated specialist teams may be asked to insert PICCs for inappropriate indications, or where there are inadequate skills or resources in the clinical teams to use alternatives. Others have reduced use of PICC catheters by improving training in ultrasound guided peripheral intravenous catheter placement. ${ }^{16}$

Fourth, to be effective on a national scale, particularly where there are wide variations in performance between individual hospitals, quality improvement programmes such as MAGIC need to be supported by robust and independent surveillance that incorporates feedback systems. ${ }^{5}$ MAGIC was introduced into an established quality improvement infrastructure and adequate staffing. Surveillance is time-consuming when reliant on manual data collection and, if resources are limited, surveillance may not be possible, or data collectors may only observe samples of the patient population. The advent of electronic patient record systems allows more patients to be monitored and in the ICU can be both more sensitive and more specific than manual collection of data for healthcareacquired infections. ${ }^{17}$ These surveillance systems are gradually improving and spreading, providing continuous feedback and peer comparison, based on semiautomated electronic data collection. ${ }^{18}$ However, the software packages required for extraction of the data are expensive and, in some countries, many hospitals do not have electronic patient records. National implementation of MAGIC could be achieved by using an existing surveillance and improvement network, or by linking local quality improvement projects, to allow peer comparisons. One such system started in the UK in 2016 and has been recruiting ICUs in surveillance of bacteraemia and catheter infections, including PICCrelated infections. ${ }^{18}$ This Infection in Critical Care Quality Improvement Programme is a joint initiative between professional organisations representing adult, paediatric and neonatal intensive care, microbiology and infection control, supported by Public Health England. In its first year, $45 \%$ of adult ICUs took part 
and reported 2.3 ICU-associated CLABSI per 1000 central venous catheter days while also demonstrating marked variation between sites in both practice and outcomes. Broadening such a programme to include other complications of PICC would be relatively straightforward.

Now is the time to see if MAGIC or similar programmes can be extended to different healthcare systems internationally. The quality of patient care may be improved considerably if the practices of the best performers were adopted more widely, whether in ensuring the appropriate means of vascular access is used and/or in improving training in insertion and care of the line. According to the context in each country, a mixture of incentives and requirements for education, surveillance and feedback of complication rates associated with PICCs, driven by leadership from professional bodies and programmes such as MAGIC, are likely to be needed for significant national improvements to be made.

Funding The authors have not declared a specific grant for this research from any funding agency in the public, commercial or not-for-profit sectors.

Competing interests None declared.

Patient consent for publication Not required.

Provenance and peer review Commissioned; internally peer reviewed.

\section{REFERENCES}

1 Fakih M, Sturm L. Paving the PICC journey: building structures, process and engagement to improve outcomes. BMJ Qual Saf 2021. doi:10.1136/bmjqs-2020-012910. [Epub ahead of print: 11 Feb 2021].

2 Krein SL, Saint S, Trautner BW, et al. Patient-reported complications related to peripherally inserted central catheters: a multicentre prospective cohort study. BMJ Qual Saf 2019;28:574-81.

3 O'Brien J, Paquet F, Lindsay R, et al. Insertion of PICCs with minimum number of lumens reduces complications and costs. $J$ Am Coll Radiol 2013;10:864-8.

4 Pronovost P, Needham D, Berenholtz S, et al. An intervention to decrease catheter-related bloodstream infections in the ICU. N Engl J Med 2006;355:2725-32.

5 Bion J, Richardson A, Hibbert P, et al. 'Matching Michigan': a 2-year stepped interventional programme to minimise central venous catheter-blood stream infections in intensive care units in England. BMJ Qual Saf 2013;22:110-23.

6 Velasquez Reyes DC, Bloomer M, Morphet J. Prevention of central venous line associated bloodstream infections in adult intensive care units: a systematic review. Intensive Crit Care Nurs 2017;43:12-22.

7 Myers JS, Wong BM. Measuring outcomes in quality improvement education: success is in the eye of the beholder. BMJ Qual Saf 2019;28:345-8.

8 Warren DK, Zack JE, Mayfield JL, et al. The effect of an education program on the incidence of central venous catheterassociated bloodstream infection in a medical ICU. Chest 2004;126:1612-8.

9 Chaiyakulsil C, Pharadornuwat O. Can central venous access device care bundles and regular feedback reduce central lineassociated complications in pediatric patients? Clin Exp Pediatr 2021;64:123-9.

10 Chopra V, Flanders SA, Saint S, et al. The Michigan appropriateness guide for intravenous catheters (MAGIC): results from a Multispecialty panel using the RAND/UCLA appropriateness method. Ann Intern Med 2015;163:S1-40.

11 Swaminathan L, Flanders S, Rogers M, et al. Improving PICC use and outcomes in hospitalised patients: an interrupted time series study using MAGIC criteria. BMJ Qual Saf 2018;27:271-8.

12 Chopra V, O'Malley M, Horowitz J, et al. Improving peripherally inserted central catheter appropriateness and reducing device-related complications: a quasiexperimental study in 52 Michigan hospitals. BMJ Qual Saf 2021. doi:10.1136/bmjqs-2021-013015. [Epub ahead of print: 29 Mar 2021].

13 Mohapatra S, Kapil A, Suri A, et al. Impact of continuous education and training in reduction of central line-associated bloodstream infection in neurointensive care unit. Indian J Crit Care Med 2020;24:414-7.

14 Inchingolo R, Pasciuto G, Magnini D, et al. Educational interventions alone and combined with Port protector reduce the rate of central venous catheter infection and colonization in respiratory semi-intensive care unit. BMC Infect Dis 2019;19:215.

15 Gerver SM, Mihalkova M, Bion JF, et al. Surveillance of bloodstream infections in intensive care units in England, May 2016-April 2017: epidemiology and ecology. J Hosp Infect 2020;106:1-9.

16 Galen B, Baron S, Young S, et al. Reducing peripherally inserted central catheters and midline catheters by training nurses in ultrasound-guided peripheral intravenous catheter placement. BMJ Qual Saf 2020;29:245-9.

17 de Bruin JS, Adlassnig K-P, Blacky A, et al. Effectiveness of an automated surveillance system for intensive care unit-acquired infections. J Am Med Inform Assoc 2013;20:369-72.

18 Schreiber PW, Dunic M, Wolfensberger A, et al. Seasonal differences in central line-associated bloodstream infection incidence rates in a central European setting: results from prospective surveillance. Am J Infect Control 2019;47:1011-3. 